\title{
Report on the Tenth Colloquium of the Postgraduate Forum on Genetics and Society (PFGS)
}

\section{CONOR DOUGLAS}

The Postgraduate Forum on Genetics and Society (PFGS) is a fluid group of doctoral (and other) researchers, organised and run by those same researchers on a voluntary basis, which is committed to providing a collegial and supportive environment for collaboration and skills training.

While the PFGS is an active online community ${ }^{1}$ in which information and news can be discussed and exchanged, its members also meet regularly to discuss and present their ongoing research, as well as participate in workshops. From 30 of August until 1 September 2006, postgraduate researchers working in and around areas of genetics and society met for the tenth time since the inception of their network in 1998.

Despite the fact that these colloquia have traditionally been held in the UK, PFGS has members the Netherlands, Austria, Finland, Australia, USA, Denmark, Germany, Romania, Tasmania, and India. Five nations were represented at the tenth meeting, held at the University of York. Disciplinary backgrounds also vary, from sociology to biology, law, philosophy, science and technology studies and theatre; the PFGS believes strongly that issues surrounding genetics and society necessitate a multiplicity of perspectives if the subject matter is to be dealt with fairly, and if learning in these areas is to flourish.

Rather than attempt to summarise the thirty-odd papers that were presented over the three days, ${ }^{2}$ this report will consist of a reflection on the theme of the event: 'Implications and implementation - the meaning and use of our research.'

Since joining the network in 2005, I have noticed a profound interest among members in the utility or impact of their research. Some members doubt whether the wider community of genetics related researchers will be receptive to their work, while others question the notions of 'impact' or 'utility' and problematise the merits of these goals when it comes to doctoral research. Still others are seeking applications for their research findings.

Irrespective of their individual positions on these matters, the thirty-plus $\mathrm{PhD}$ students attending the colloquium were invited to address these issues head-on by way of a series of 'implementation workshops.'

\section{The goals and the realities of 'implementation workshops'}

Our original vision for these workshops was to team-up various 'users' of genetic information with PFGS members so that a connection could be made between the research that PFGS members are doing and genetic practices 'out there'. The hope was that our research and our skills might apply to their work in practice and, conversely, that their experiences with the various elements of genetics might inform our own work. 
The fact of the matter is that postgraduate research does not take place within an experiential vacuum. Arguably, this is particularly true of research in areas of genetics and society. This is because (postgraduate) research in this area often employs ethnographic research techniques, which can mean that for weeks on end we find ourselves shoulder to shoulder with lab scientists as they isolate strands of DNA or stem cells lines. During this process we evolve from being laboratory fixtures that are useful for making coffee, and become lab assistants, friends and confidants of the scientists in question.

In other doctoral research scenarios PFGS, members are engaged with in-depth qualitative research with end-users of genetic sciences (eg, patients or medical practitioners). In these situations we are not simply 'in the field collecting data,' but are interacting with real people who have lives and stories. Data cannot, therefore, be harvested as if they were a crop. Indeed, frequently, such research leads $u s$ to become a part of their stories.

As a result, PFGS members can find themselves in situation in which they are not simply observing the processes by which the content of science is created or consumed; rather, often we can find ourselves knee-deep in the production process itself or in the difficult position of mediating how this knowledge is internalised.

This level of engagement in genetics suggests that PFGS researchers (among others) are in a position to make a valuable contribution to the areas that they study. The four implementation workshops were intended to highlight these contributions to the workshop leaders - people who regularly use various kinds of genetic information.

\section{Leaders of the implementation workshops}

\section{Dr Angela Flannery, director within R\&D genetics at AstraZenica}

In a very engaging presentation Dr. Flannery 'tested' PFGS members as to their knowledge that genetics plays in the drug development pipeline. Suitably impressed with our level of knowledge of the subject matter, Dr. Flannery spoke of how 'big pharma' does indeed see a use for the kinds of research being conducted by members of the PFGS. For instance, she mentioned the need for industry to have a firmer handle on shifting licensing, ethical and regulatory procedures, as well as public engagement activities about genetic testing and levels of acceptance of genetics in drugs among patients.

\section{Dr Helen Middleton-Price, director of the North West Genetics Knowledge Park (NOWGEN)}

Dr. Middleton-Price first gave an overview of aims and activities within the North West Genetics Knowledge Park. This was followed by a discussion about "the usual misunderstandings" and challenges of working in an interdisciplinary environment. For example, many bioscientists and medical professionals feel that the contribution of social scientists to their work is limited to helping out with ethical approval forms, while social scientists in turn feel they have a monopoly on studying issues to do with socio-economic aspects of innovative health technologies and access to patient care and treatment. 
Miss. Pam Davies, family services manager at the patient advocacy group CLIMB Miss. Davies discussed the role of 'information provision' that CLIMB plays for families with children with rare chromosomal disorders. She also detailed its role in funding research into these disorders. Discussion centred on the role CLIMB played in pseudo-diagnosing children who came into contact with their organisation, and the professional implications that pseudo-diagnosis and information provision has for the medical community.

Mrs. Tara Clancy, consultant genetic counsellor and lecturer in medical genetics, St. Mary's Hospital, Manchester

This interactive session stimulated discussion between attendees and Mrs. Clancy regarding the differences between what genetic counsellors do in theory, and what actually happens in practice. Along these lines, conditions and contexts in which counselling takes place were discussed and attendees were able to scope future areas that may pose new or different ethical issues. The discussion concluded with critical reflection on the ability of biomedical ethics to deal with socio-political issues that advances in biosciences are now beginning to pose.

\section{The problem of 'implementation'}

Our original vision for these implementation workshops - to promote dialogue about the use of genetic information between doctoral researchers and actors outside of academia - may have been overly ambitious for an afternoon of meetings. In retrospect, without the workshop leaders doing a lot of background reading on the subject of our dissertations, and without the doctoral researchers familiarising themselves with the role and function of these 'users' of genetic information, such links would be difficult to achieve in the 90 minute workshops. Nevertheless, these were extremely interesting sessions. PFGS members certainly benefited from the discussions about how genetic information is used in practice, and I believe that new ideas and avenues of research were spawned during these sessions. After presentations from the 'users' of genetic information, I feel that PFGS members were able to identify where their research could apply in practice. Perhaps similar presentations from PFGS members about their own research would have fostered further collaboration, in that the 'users' could then see for themselves where our work could fit into their practices.

Would presentations be enough to foster collaboration and lead to the implementation of our work? Is this implementation the goal of $\mathrm{PhD}$ research? If the York workshops did not lead to direct collaboration, they certainly provided a platform for reflection on questions such as these.

After these workshops, informal discussion centred on the notion of change (ie, the consequence of implementation) and how this notion can be much more nuanced and subtle than the traditional 'cause and effect' framework that is applied in much of the science we study. Can doctoral students expect to see direct results from our research efforts, even if we are lucky enough to make collaborative links with users of genetic information outside of academia? If so, what might those results look like? What form would they take? How would we be able to judge or assess the relative impacts of 
these results? All of these questions highlight the complexities faced by the doctoral students at the York meeting.

Practical problems associated with interdisciplinary research were also raised. Can implementation take place when professional and disciplinary boundaries are constantly being reproduced and defended by those we are attempting to work with? Such questions were not directed at the leaders of the workshops, but simply reflected the issue of power and complexity in knowledge production and dissemination.

Members certainly felt that there was great value and potential in working across disciplinary and professional boundaries. If we could collectively get as far as learning (that is two-way learning... we learn from them and they learn from us), then that would represent a great start.

\section{Closing remarks: the importance of postgraduate research and its implementation}

What will be the result of years of research and writing that is represented in our theses?

What are the possible areas in which it might make an impact?

How can our research be informed by differing applications of genetic information in practices?

What are examples of genetics in practice, and how might our research make in impact in those areas?

Irrespective of the relative success or failure of the implementation workshops, I would maintain that the questions above are (or dare I say "should be") central concerns for doctoral research being undertaken in the arena of genetics and society. The issues examined by PFGS members (along with other doctoral research) are simply too important to be left in academic ivory towers, and we spend simply too much time on the work to let it evaporate into obscurity.

It is not simply the effort that is invested in these projects undertaken by PFGS members that makes them important, however; they also have the potential to add real value to the production of genetic sciences. As we stand shoulder to shoulder with laboratory researchers, or share a cup of tea with a patient with a genetic disorder, we straddle the boundary between the often very different worlds of science and society. By doing so we are in a rare position to create links between the knowledge production process and its subsequent applications. Furthermore, while we are lucky enough to have access rights to these knowledge production and consumption processes, I feel that we have a concurrent responsibility to bring a certain amount of reflexive engagement to both of these activities. The production and consumption of genetic sciences are not passive activities. The degree to which co-production processes are at work perhaps even call into question such linear terms as 'production' and 'consumption'. Pains must be taken to make this clear when implementation is 
sought, and we should not be satisfied with a hope that the messages and value within doctoral research falls on sympathetic ears.

Clearly, there are challenges to linking up academic work with day-to-day practices in genetics. Perhaps this is true of doctoral research in particular as we often lack the institutional longevity, publication record, or network associations of other academics. Nevertheless, with some of the challenges of implementation now on the radar for the PFGS surely we are in a better position to open up productive dialogues between these disparate actors working in related areas.

In some research careers, the opportunity to delve as deeply into a substantive area as by doctoral work permits rarely presents itself again. Seldom does a research topic get such focused and undivided attention as when a $\mathrm{PhD}$ project is applied to it. These projects represent a great opportunity, not only for the researcher but also for potentially positive change to the associated area of inquiry. It would be a shame to see these opportunities misspent. Recognition of the value of doctoral researcher is required, but at the same time onus also rests on those doctoral researchers to step outside of their comfort zone and do something with their work. This represents a challenge indeed, but a rewarding one surely worth the effort.

\section{Acknowledgements}

The PFGS warmly thank the ESRC Genomic Policy and Research Forum for its support of our network for the third consecutive year. We also thank the ESRC Innovative Health Technologies Programme for its support in 2006 as well as in 2002.

\footnotetext{
${ }^{1}$ The PFGS manages a website at http://pfgs.org/ and a bulletin board at http://pfgs.org/phpBB/index.php. The website contains information on previous colloquia, a newsletter, a peer-review journal, and details of upcoming events including our own regional meetings and workshops. The bulletin board is a site for direct discussion of issues of interest to researchers working in the field of genetics and society. To become a member of the PFGS please visit the website or contact the author of this paper. Membership is free.

${ }^{2}$ Abstracts of papers, the colloquium programme and more can be viewed at http://pfgs.org/mambo/index.php?option=com content\&task=category\&sectionid=7\&id=46\&Itemid=4 9. Sessions included: public understanding and participation of genetics; international governance in bioscience; DNA sequencing and standardization; genetic privacy \& law; tissues \& issues; negotiation and expectation in bioscience; implementing genetics into clinical practice; and the future of science $\&$ biocapital.
} 